\title{
Trans-cyclopropanation of mycolic acids on trehalose dimycolate suppresses Mycobacterium tuberculosis-induced inflammation and virulence
}

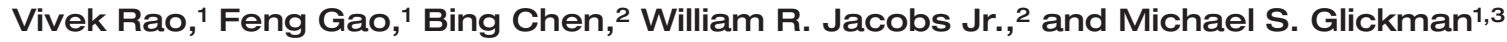 \\ 1Immunology Program, Sloan-Kettering Institute, Memorial Sloan-Kettering Cancer Center, New York, New York, USA. \\ ${ }^{2}$ Howard Hughes Medical Institute, Department of Microbiology and Immunology, Albert Einstein College of Medicine, New York, New York, USA. \\ ${ }^{3}$ Division of Infectious Diseases, Memorial Sloan-Kettering Cancer Center, New York, New York, USA.
}

\begin{abstract}
Recent studies have shown that fine structural modifications of Mycobacterium tuberculosis cell envelope lipids mediate host cell immune activation during infection. One such alteration in lipid structure is cis-cyclopropane modification of the mycolic acids on trehalose dimycolate (TDM) mediated by proximal cyclopropane synthase of $\alpha$ mycolates (pcaA), a proinflammatory lipid modification during early infection. Here we examine the pathogenetic role and immunomodulatory function of mycolic acid cyclopropane stereochemistry by characterizing an M. tuberculosis cyclopropane-mycolic acid synthase 2 (cmaA2) null mutant $(\triangle c m a A 2)$ that lacks trans-cyclopropanation of mycolic acids. Although titers of WT and $\triangle c m a A 2$ organisms were identical during mouse infection, $\triangle \mathrm{cmaA2}$ bacteria were hypervirulent while inducing larger granulomas than WT M. tuberculosis. The hypervirulence of the $\triangle c m a A 2$ strain depended on host TNF- $\alpha$ and IFN- $\gamma$. Loss of trans-cyclopropanation enhanced $M$. tuberculosis-induced macrophage inflammatory responses, a phenotype that was transferable with petroleum ether extractable lipids. Finally, purified TDM lacking trans-cyclopropane rings was 5-fold more potent in stimulating macrophages. These results establish $\mathrm{cmaA2}$-dependent trans-cyclopropanation of TDM as a suppressor of M. tuberculosis-induced inflammation and virulence. In addition, cyclopropane stereochemistries on mycolic acids interact directly with host cells to both positively and negatively influence host innate immune activation.
\end{abstract}

\section{Introduction}

The ongoing global health crisis of tuberculosis infection has stimulated intense investigation into the molecular basis of Mycobacterium tuberculosis pathogenicity (1-5). A substantial subset of genes identified as important for $M$. tuberculosis pathogenesis is involved in biosynthesis of the complex mycobacterial cell envelope. The mycobacterial cell envelope is a complex array of lipids and glycolipids that forms a hydrophobic barrier but also contains substantial adjuvant activity (6-8). Several cell wall determinants implicated in M. tuberculosis pathogenesis include phthiocerol dimycocerosate, phenolic glycolipid (PGL), oxygenated mycolates, mycolic acid length, and mycolic acid cyclopropanation (9-15). While some of these lipids appear to mediate pathogenesis through their contribution to a hydrophobic barrier (16), recent evidence strongly supports a model in which specific $M$. tuberculosis cell envelope lipids directly activate or repress host innate immune responses.

One such lipid is PGL, a glycolipid produced universally by Mycobacterium leprae but sporadically by M. tuberculosis strains (17). Recently, a globally disseminated clinical strain was shown to be hypervirulent for mice. This strain synthesized PGL due to the absence of a frameshift mutation in the pks1-15 genes present in many TB strains (15). Purified PGL directly inhibited TNF- $\alpha$

Nonstandard abbreviations used: $c m a A 2$, cyclopropane-mycolic acid synthase 2; pcaA, proximal cyclopropane synthase of $\alpha$ mycolates; PGL, phenolic glycolipid; TDM, trehalose dimycolate.

Conflict of interest: The authors have declared that no conflict of interest exists. Citation for this article: J. Clin. Invest. 116:1660-1667 (2006). doi:10.1172/JCI27335. secretion from macrophages, suggesting that PGL in the cell envelope of these strains promoted hypervirulence through suppression of immune activation. Similarly, our recent study with a mutant strain of M. tuberculosis deficient in cis-cyclopropanation of the $\alpha$ mycolic acid demonstrated that this lipid modification was required for full activation of innate immune responses during early infection (18). This immunostimulatory effect of cyclopropanation was due to altered inflammatory activity of trehalose dimycolate (TDM), a long-suspected glycolipid virulence determinant of M. tuberculosis and related pathogenic mycobacteria (19-26). These results implicated cis-cyclopropanation of TDM as a proinflammatory lipid modification. Taken together, these 2 examples validated the hypothesis that the M. tuberculosis cell envelope is in part a complicated array of lipid effector molecules that interact with host cells (27).

In addition to proximal cyclopropane synthase of $\alpha$ mycolates (pcaA)mediated cis-cyclopropanation of mycolic acids, M. tuberculosis expresses a family of cyclopropane synthases that modify mycolic acids at specific locations or with specific stereochemistries of cyclopropane rings (28-32). Genetic deletion of cyclopropane-mycolic acid synthase 2 (cmaA2) abolishes trans-cyclopropanation of both methoxyand ketomycolates while $\alpha$ mycolates are unaffected (30). While ciscyclopropanation of membrane fatty acids is widely distributed in bacteria and plants (33-36), trans-cyclopropane rings are exceedingly rare. Here we examine the biological function of trans-cyclopropanation in M. tuberculosis and show that the trans-cyclopropanation of mycolic acids on TDM limits M. tuberculosis virulence and suppresses host inflammatory responses during infection. 


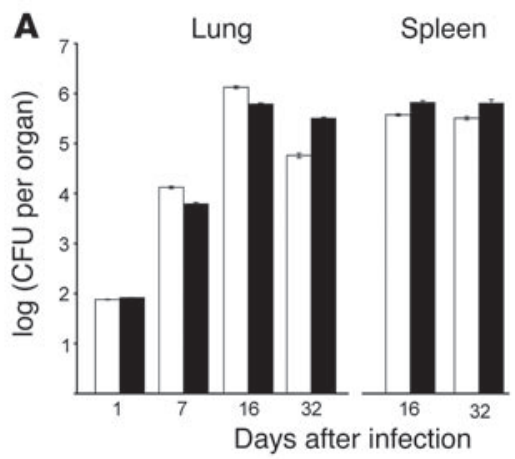

C

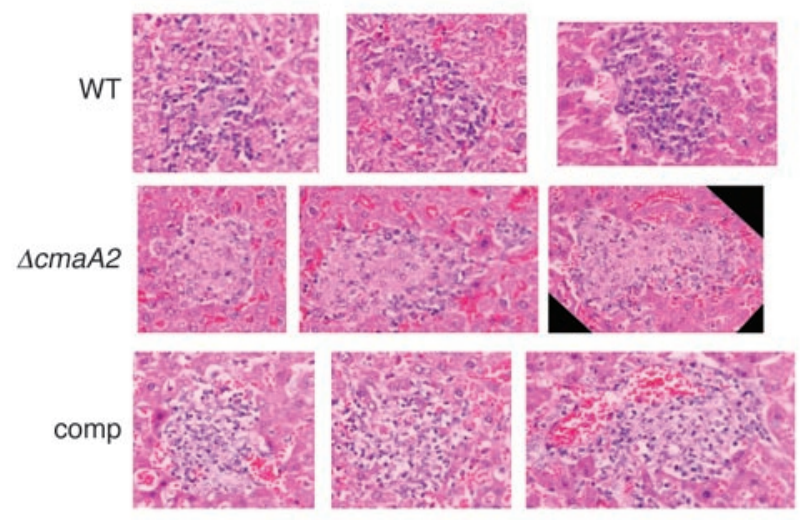

B

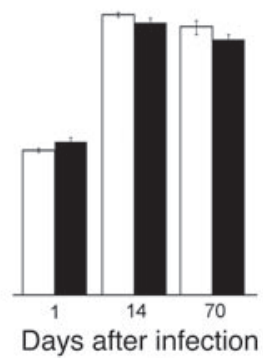

D

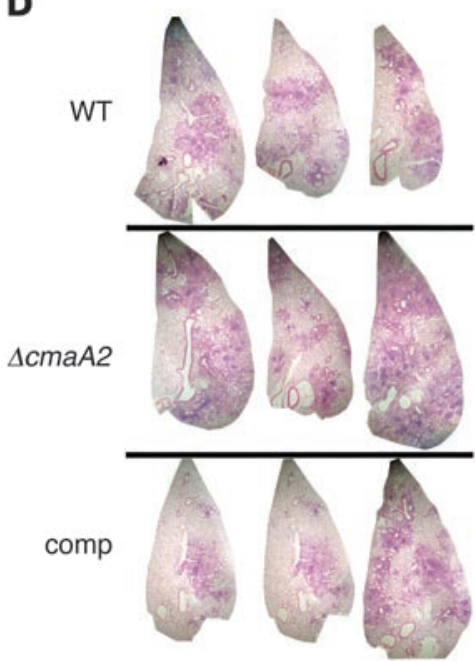

\section{Figure 1}

Loss of cmaA2 does not affect bacterial number but alters granuloma composition. (A) WT C57BL/6J mice were infected by aerosol with WT $M$. tuberculosis (white bars) or the $\triangle c m a A 2$ mutant (black bars), and bacterial titers in lungs and spleen were determined at the indicated time points by serial dilution and plating of tissue homogenates. Each bar shows the mean value of bacterial loads from 3 mice per group with error bars indicating SEM. (B) Bacterial loads in lungs of animals infected intravenously with WT M. tuberculosis (white bars) or the $\triangle c m a A 2$ mutant (black bars). (C) Altered granuloma structure in tissues upon infection with $\triangle c m a A 2$. Representative liver granulomas (3 per infecting strain) from mice infected with WT, $\triangle c m a A 2$, and complemented (comp) strain. Magnification, $\times 400$. See Results for statistical analysis of granuloma size and cellular composition. (D) H\&E-stained lungs from C57BL/6J mice infected with the indicated strains and sacrificed 6 months after infection. See Results for quantitation of granulomatous inflammatory lesions.

\section{Results}

cmaA2 suppresses $M$. tuberculosis virulence. To understand the pathogenetic role of mycolic acid trans-cyclopropanation in $M$. tuberculosis, we characterized an $M$. tuberculosis cmaA2 null mutant that lacks transcyclopropanation in all oxygenated mycolic acids but synthesizes intact ratios of all mycolic acid subclasses (30). We infected C57BL/6J and BALB/C mice with WT M. tuberculosis, a cmaA2 null mutant, and a genetically complemented cmaA2 mutant carrying a single copy of WT $c$ maA2 expressed from its native promoter. Both BALB/C and C57BL/6J mice received either 100 CFUs by aerosol or $10^{5}$ or $10^{6}$ CFUs by tail vein injection. Regardless of infection route or mouse strain, loss of $c m a A 2$ did not affect bacterial loads in lungs, liver, and spleen during the intial 70 days of infection (Figure 1, A and B, and data not shown). These results demonstrate that loss of $\mathrm{cmaA} 2$ function in M. tuberculosis is not important for bacterial colonization, replication, or persistence in vivo during the first 70 days of infection.

Many recent studies demonstrated that $M$. tuberculosis virulence and immunopathology can occur independently of bacterial titer mation than WT lungs (Figure 1D): $38 \%$ of WT lung area was replaced by inflammatory lesions while $\Delta c m a A 2$-infected lungs displayed 52\% involvement and complemented mutant infected lungs displayed $24 \%$ involvement. These results indicate that inactivation of $c m a A 2$ results in a pathologically more aggressive infection both early and late in infection.

To ask whether these differences in granuloma composition reflected changes in strain virulence, we examined host morbidity in infected mice after intravenous and aerosol infection. $\Delta c m a A 2$ $M$. tuberculosis-infected mice succumbed to infection earlier than WT infected mice in both intravenous infection of BALB/C (Figure 2A) and $\mathrm{C} 57 \mathrm{BL} / 6 \mathrm{~J}$ (Figure 2B) mice and in aerosol infection of C57BL/6J mice (Figure 2C). The mean survival time of C57BL/6J mice infected with $10^{5} \mathrm{CFUs}$ of WT or $\triangle c m a A 2$ strains was 320 and 227 days, respectively $(P<0.0001)$ (Figure $2 \mathrm{~B})$. BALB/C mice infected with $10^{6}$ of WT, $\triangle c m a A 2$, or the complemented mutant had mean survival times of 113,90 , and 116 days, respectively $(P<0.0001$ for WT versus mutant; $P=0.003$ for complemented 
A

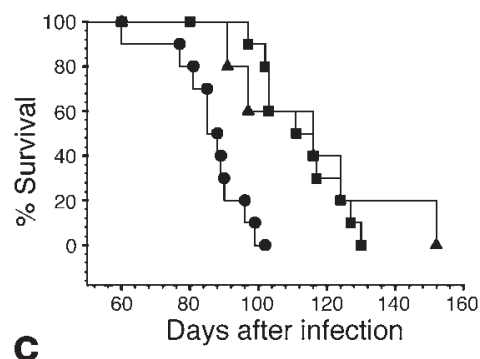

C

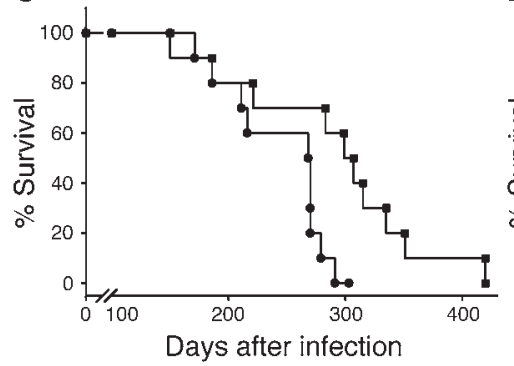

B
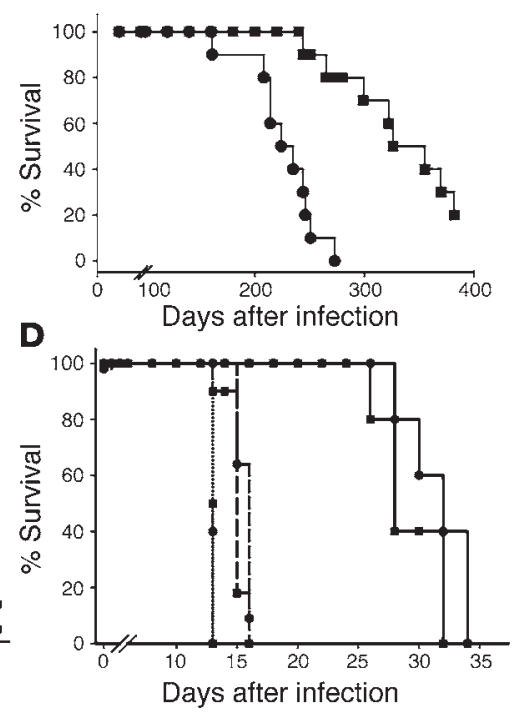

Figure 2

cmaA2 suppresses $M$. tuberculosis virulence in mice. (A) Survival kinetics of WT BALB/C mice infected via tail vein injection with $10^{6} \mathrm{CFU}$ s of either WT M. tuberculosis (squares), $\triangle c m a A 2 M$. tuberculosis (circles), or the complemented $\triangle c m a A 2$ strain (triangles). (B and C) Survival kinetics of WT C57BL/6J mice infected with $M$. tuberculosis (squares) or $\triangle c m a A 2 M$. tuberculosis (circles) by tail vein injection $\left(10^{5}\right.$ CFUs, B) or aerosol (100 CFUs, C). (D) Survival of immunodeficient mice infected with WT (squares) and $\triangle c m a A 2$ (circles) strains. TNF- $\alpha$-deficient mice (solid lines), IFN- $\gamma$-deficient mice (dotted lines), and SCID mice (dashed lines) were infected as described in Methods, and survival was recorded. versus mutant; $P=0.37$ for WT versus complemented; Figure $2 \mathrm{~A}$ ). Mean survival times of C57BL/6J mice infected with 100 CFUs of WT M. tuberculosis was 314 days and 247 days for $\triangle c m a A 2$ mutant infected mice $(P=0.0002)$ (median survival: WT 318 days; $c m a A 2$, 263 days). Taken together, these results demonstrate that the $\mathrm{cmaA2}$ gene and trans-cyclopropanation of cell envelope mycolic acids restrain $M$. tuberculosis virulence and alter granuloma composition.

Requirement of host immunity for the hypervirulence of the $\triangle c m a A 2$ strain. Earlier work from several research groups implicated cellmediated immunity and cytokines such as TNF- $\alpha$ and IFN- $\gamma$ as important mediators of host defense against $M$. tuberculosis infection in mice and humans (42-46). Our previous results with the M. tuberculosis $\triangle p c a A$ mutant demonstrated that early induction of TNF- $\alpha$ in host macrophages is affected by the cis-cyclopropane content of mycolic acids on TDM. To test the role of TNF- $\alpha$ in the hypervirulent phenotype of the $\mathrm{cmaA} 2$ mutant, we infected TNF- $\alpha^{-/}$ mice by aerosol and recorded host morbidity. TNF- $\alpha$-deficient mice succumbed to infection with either strain in approximately 36 days, as reported previously for $M$. tuberculosis aerosol infection (47). In contrast to increased susceptibility of WT mice to $\triangle c m a A 2$ infection, there were no differences in mortality of TNF- $\alpha$ deficient mice upon infection with either strain (Figure 2D). Similar kinetics of host susceptibility were also observed in both IFN- $\gamma$-deficient and SCID mice (Figure 2D) when either strain was administered intravenously (48). Thus, increased virulence of $\triangle c m a A 2$ mutant in WT mice depends on TNF- $\alpha$, IFN- $\gamma$, and intact lymphocyte populations. These results imply that the hypervirulence of the cmaA2 mutant strain is a manifestation of excessive immune activation, which produces larger and altered granulomas and, ultimately, premature death.

\section{Figure 3}

$\triangle$ cmaA2 M. tuberculosis is hyperinflammatory during macrophage infection. RAW 264.7 macrophages $(\mathbf{A})$, BALB/C murine bone marrow-derived macrophages (B), and C57BL/6J bone marrow-derived macrophages $(\mathbf{C})$ were left uninfected (light gray bars) or infected with WT M. tuberculosis (white bars), $\triangle c m a A 2$ mutant $M$. tuberculosis (black bars), or the complemented $\triangle c m a A 2$ mutant (dark gray bars). At the indicated time points after infection, TNF- $\alpha$ levels in culture supernatants were determined by ELISA. Values $(\mathrm{pg} / \mathrm{ml})$ indicate mean concentration of TNF- $\alpha$ in triplicate infections, and levels of statistical significance by 2-tailed Student's $t$ test are indicated above the bars.
A
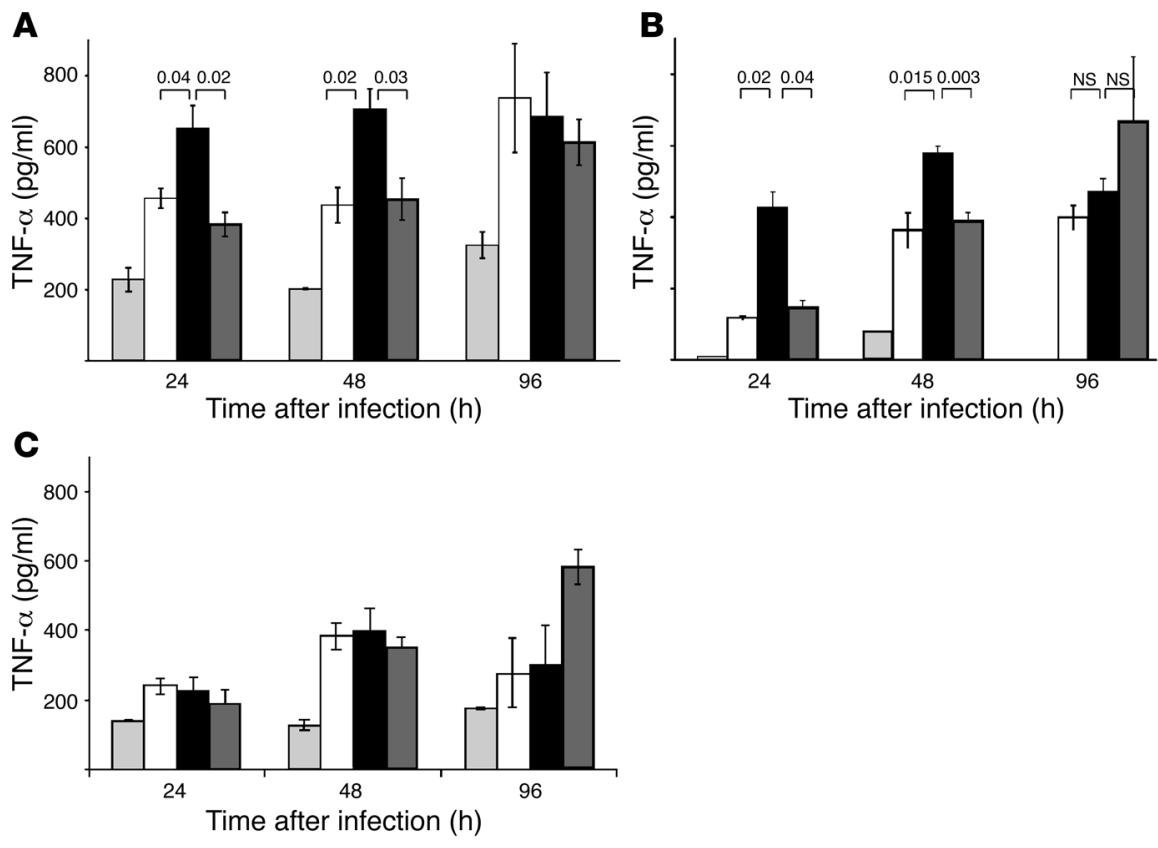


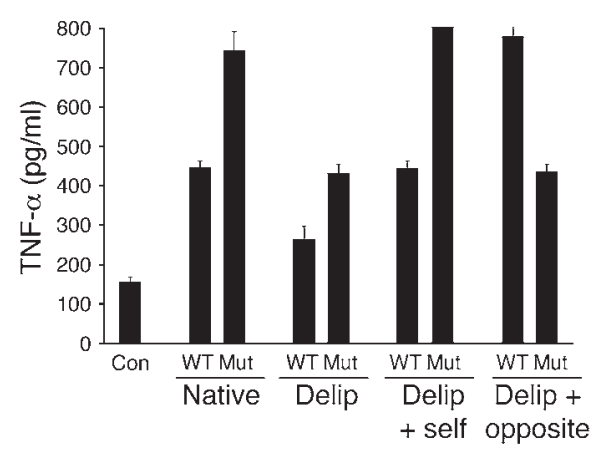

\section{Figure 4}

cmaA2-dependent extractable lipid modification suppresses $M$. tuberculosis-induced macrophage activation. Bone marrow-derived macrophages from BALB/C mice were left uninfected (con), or infected with WT M. tuberculosis or $\triangle c m a A 2$ mutant $M$. tuberculosis (native), delipidated bacteria (delip), delipidated bacteria reconstituted with self lipids (delip + self), or delipidated and reconstituted with lipids from the opposite strain (delip + opposite). TNF- $\alpha$ levels were determined 24 hours after infection. Values are mean concentrations of quadruplicate wells.

Early increase in macrophage cytokine responses to $\triangle c m a A 2 M$. tuberculosis. Multiple prior studies documented complicated relationships between cytokine induction and granuloma structure. To test whether the phenotype of the $\triangle c m a A 2$ strain is associated with altered proinflammatory cytokine release from macrophages, we infected RAW 264.7 macrophages and murine bone marrowderived macrophages and measured TNF- $\alpha$ release. As early as 24 hours after infection, $c m a A 2$-deficient bacilli stimulated significantly higher levels of TNF- $\alpha$ from both RAW and BALB/C bone marrow-derived macrophages (Figure 3 , A and B) but not from C57BL6J bone marrow-derived macrophages (Figure 3C). The hyperinflammatory phenotype of the $\triangle c m a A 2$ strain was reversed by genetic complementation by a functional $\mathrm{cmaA} 2$ gene (Figure 3, $A$ and $B$ ), demonstrating that the phenotype of the mutant strain was due to loss of $c m a A 2$ function. Thus, inactivation of $c m a A 2$ in M. tuberculosis causes hyperinflammation during infection of macrophages, which is temporally restricted to early infection.

Cyclopropane-deficient extractable lipids directly mediate the byperinflammatory phenotype of cmaA2 mutant bacteria. Based on our prior results with the pcaA mutant, we hypothesized that the trans-cyclopropane modification of extractable lipids in the cell envelope negatively influenced macrophage activation. To analyze the contribution of extractable lipids to the $c$ maA2 mutant phenotype, we performed a lipid transfer experiment. Petroleum ether delipidation does not kill $M$. tuberculosis and removes a limit set of extractable lipids that is more than 90\% TDM when examined by TLC (18). Native $\triangle c m a A 2$ bacilli induced approximately 2-fold higher levels of TNF- $\alpha$ than WT bacteria (Figure 4; $P<0.001$ ). Delipidation reduced the inflammatory capacity of $\triangle c m a A 2$ bacilli to WT levels (Figure 4). Reconstitution of the delipidated bacteria with their corresponding lipid extracts restored the native phenotypes (Figure 4). However, when delipidated WT bacteria were reconstituted with $\triangle c m a A 2$ lipids, the hyperinflammatory phenotype of the $\triangle c m a A 2$ strain was transferred (Figure 4), demonstrating that the hyperinflammatory phenotype was directly attributable to the extractable lipid fraction of $M$. tuberculosis. Conversely, $\Delta c m a A 2$ mutant bacteria acquired the WT inflammatory phenotype when reconstituted with WT lipids.
Trans-cyclopropane-deficient TDM is hyperinflammatory. TDM is an inflammatory glycolipid of the $M$. tuberculosis cell envelope that contains 2 cyclopropanated mycolic acids. Our prior work demonstrated that TDM from the pcaA mutant strain of $M$. tuberculosis is hypoinflammatory for macrophages and mice. We hypothesized that the hyperinflammatory phenotype of the $\triangle c m a A 2$ mutant could be due to altered inflammatory potency of $\triangle c m a A 2$-derived TDM. To test this idea, we purified TDM from WT M. tuberculosis, $\Delta c m a A 2$, and the complemented strains. All 3 preparations were chemically pure when examined by thin layer chromatography and comigrated with commercially available TDM from M. tuberculosis, indicating their identity and homogeneity (data not shown).

We stimulated macrophages in vitro with monolayers of different concentrations of purified TDM from the 3 strains and measured TNF- $\alpha$ secretion. Dose-response curves derived from these experiments indicated that $\triangle c m a A 2$ mutant-derived TDM was 4- to 6-fold more potent than either WT TDM or complemented mutant TDM, as assessed by the quantity of glycolipid to achieve half-maximal TNF- $\alpha$ release or the fold increase in TNF- $\alpha$ release at a fixed dose of TDM (Figure 5A). We did not observe any difference between WT and $\Delta \mathrm{cmaA} 2$ TDM in TNF- $\alpha$ induction from C57BL/6J-derived bone marrow macrophages (data not shown). These results implicate the trans-cyclopro-
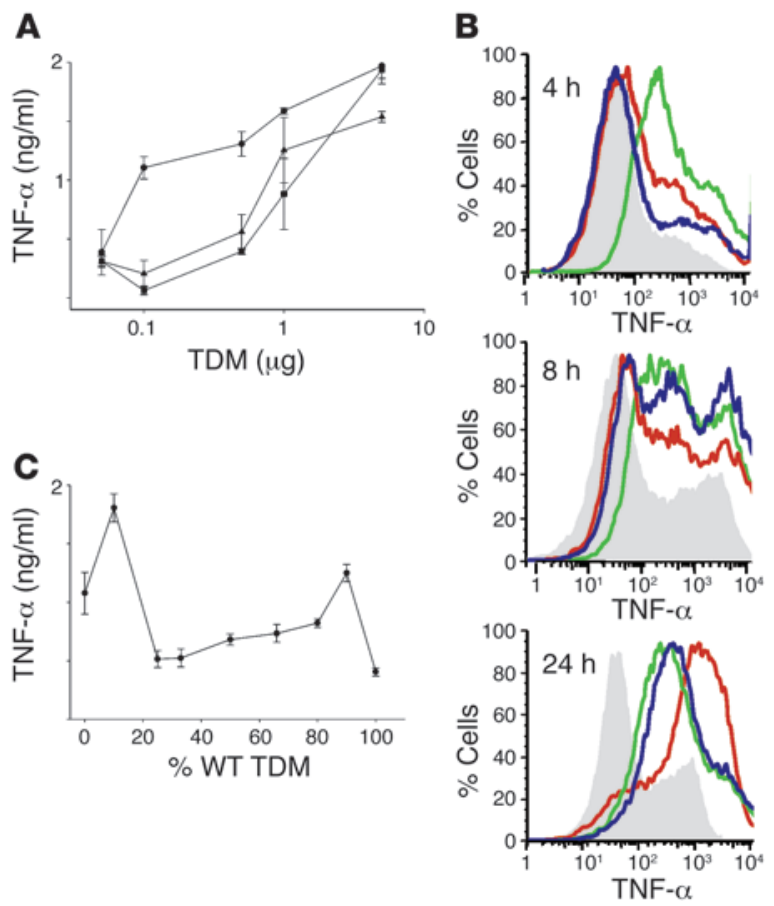

\section{Figure 5}

Trans-cyclopropane-deficient TDM is hyperinflammatory for macrophages. (A) Dose-response curve for TNF- $\alpha$ induction by WT TDM (squares), $\triangle c m a A 2$ mutant TDM (circles), or $\triangle c m a A 2$ mutant complemented TDM (triangles) from RAW cells measured after 24 hours of stimulation. (B) RAW 264.7 cells were stimulated with vehicle (gray areas), WT TDM (red lines), $\triangle c m a A 2$ mutant TDM (green lines), or complemented TDM (blue lines) for 3 hours, and single cell levels of TNF- $\alpha$ were determined by intracellular cytokine staining for TNF- $\alpha$. (C) Macrophages were stimulated with a total TDM dose of $1 \mu \mathrm{g}$ in varying ratios of WT/ $\triangle c m a A 2$ TDM as indicated, and TNF- $\alpha$ in culture supernatants was measured by ELISA 6 hours after stimulation. 
pane ring on the mycolates of TDM as a negative regulator of the inflammatory potency of this glycolipid.

To more completely characterize the temporal induction of TNF- $\alpha$ by these TDMs, we quantitated single-cell TNF- $\alpha$ production by macrophages using intracellular cytokine staining and flow cytometric analysis. Interestingly, $\triangle c m a A 2$ TDM induced very high levels of TNF- $\alpha$ at as early as 4 hours of stimulation in comparison with the low levels of induction by the WT TDM (Figure 5B). Enhanced TNF- $\alpha$ induction by the mutant TDM was also maintained after 8 hours of stimulation. However, by 24 hours of stimulation, the level of TNF- $\alpha$ induction was significantly higher for WT TDM in comparison with the $\triangle c m a A 2$ TDM. These quantitative and kinetic differences in TNF- $\alpha$ induction from macrophage cultures were overcome by complementation of the $\triangle c m a A 2$ mutant with a functional $c m a A 2$ gene (Figure $5 \mathrm{~B}$ ).

The hyperinflammatory properties of $\triangle c m a A 2$ TDM could be due either to loss of an inhibitory effect of the trans-cyclopropane ring in WT TDM or an enhanced stimulatory property of $\triangle c m a A 2$ mutant TDM. If the trans-cyclopropane ring on TDM is directly inhibitory, this property should suppress the hyperinflammatory effect of trans-cyclopropane-deficient TDM. Alternatively, if trans-cyclopropane-deficient TDM is a stronger proinflammatory agonist, then combination of WT and mutant TDMs should be additive or indifferent but not antagonistic. To distinguish these possibilities, we stimulated macrophages with WT, $\triangle c m a A 2$, or varying ratios of both TDMs, holding the total dose of TDM constant, and analyzed TNF- $\alpha$ secretion. At WT/ $\Delta c m a A 2$ ratios of $0.2: 0.8,0.4: 0.6$, and $0.5: 0.5$, the hyperinflammatory properties of the cmaA2 TDM were inhibited by the WT TDM (Figure 5C). These results suggest that the trans-cyclopropane ring in WT TDM is an inhibitor of macrophage activation.

\section{Discussion}

In this study, we examined the pathogenetic role of trans-cyclopropanation of mycolic acids in the M. tuberculosis cell envelope. We have shown that trans-cyclopropanation is a negative regulator of $M$. tuberculosis virulence through suppression of the inflammatory activity of TDM. These results define a novel role for the rare chemical entity of trans-cyclopropane rings and expand the diversity of pathogenic functions mediated directly by cell envelope lipids of $M$. tuberculosis.

cmaA2 suppresses $M$. tuberculosis virulence. The traditional definition of virulence genes is a genetic determinant that enhances pathogenesis. However, there is growing recognition that pathogens that cause persistent infections employ complex strategies of pathogenesis, including functions that limit pathogen virulence or host pathology. Several genetic loci in M. tuberculosis are known to restrict virulence and, when mutated, result in hypervirulence in SCID mice $(49,50)$. In immunocompetent mice, inactivation of the mceI operon in M. tuberculosis resulted in a hypervirulent strain that grew to higher bacterial loads and caused accelerated host mortality (51). The mechanism by which these genetic loci restrict M. tuberculosis virulence is not clear.

Recent work demonstrated that some clinical strains of M. tuberculosis are hypervirulent for mice and produce PGL through preservation of an intact $p k s 1 / 15$ gene (15). This hypervirulence was associated with suppression of macrophage inflammatory responses by PGL, suggesting that this glycolipid directly suppresses innate immune activation to promote bacterial virulence. The present study defines $c m a A 2$ as a negative regulator of $M$. tuberculosis viru- lence and provides insight into the mechanism of $c m a A 2$-dependent attenuation. Trans-cyclopropanation of mycolic acids on TDM restricted $M$. tuberculosis-induced macrophage inflammation during early infection, thereby altering granuloma structure and ultimately restricting virulence. The results presented here, taken together with prior results with PGL and the $\Delta p c a A$ mutant of $M$. tuberculosis, define 3 lipids that dynamically regulate macrophage activation during early infection and highlight the complex relationship among the mycobacterial cell envelope, innate immune activation, and $M$. tuberculosis virulence. While the $\triangle c m a A 2$ mutant and the HN878 strain share hypervirulence in the mouse, they differ in their inflammatory phenotypes for macrophages, 1 being hyperinflammatory $(\triangle c m a A 2)$ and 1 being hypoinflammatory (HN878). These results are consistent with abundant prior literature demonstrating that appropriate timing and levels of TNF- $\alpha$ are critical in preventing uncontrolled infection (TNF- $\alpha$ deficiency) or excessive tissue destruction (TNF- $\alpha$ excess) and that $M$. tuberculosis actively represses host macrophage activation (52-55).

Although the hypervirulent phenotype of the $\triangle c m a A 2$ strain was present in both $\mathrm{C} 57 \mathrm{BL} / 6 \mathrm{~J}$ and BALB/C mice, the hyperstimulatory phenotype for macrophages was only observed in BALB/C-derived cells. This may indicate that the in vivo source of TNF- $\alpha$ that mediates hypervirulence is a cell type other than macrophages. There is evidence that $\mathrm{T}$ cell- and macrophage-derived TNF- $\alpha$ play distinct roles in the containment of $M$. tuberculosis infection and that T cell-derived TNF- $\alpha$ modifies the granulomatous pathology of M. tuberculosis infection (56).

Cyclopropanation of bacterial lipids. Our recent work defines an immunomodulatory function for cyclopropanation of bacterial membrane lipids in the context of microbial pathogenesis. The most extensively studied cyclopropane modification system in bacteria is the CFA synthase (CFAS) of Escherichia coli, which ciscyclopropanates membrane fatty acids during entry into stationary phase. E. coli deficient for CFAS is sensitive to acid (57), but no role in bacterial pathogenesis has been recognized. An extensive system of S-adenosyl methionine-dependent cyclopropane synthases exists in $M$. tuberculosis, and each enzyme is able to modify mycolic acids with a specific stereochemistry of cyclopropyl group or at a specific location. Although these cyclopropane modifications may indeed play a structural role in the integrity of the cell envelope, evidence presented here and our prior work implicate 2 stereochemistries of cyclopropane rings in pathogen-induced immunomodulation. Such a role is supported by recent studies examining the M. tuberculosis transcriptional profile while growing in human lung (58). This study found in vivo induction and repression of mycolic acid cyclopropane synthases, including pcaA, $c m a A 2, m m a A 2$, and $m m a A 1$, supporting the idea that M. tuberculosis modifies its cell envelope in response to the host. Further studies of a complete set of mycolic acid cyclopropane synthase mutants in M. tuberculosis will further define the functions of this unique lipid modification.

Innate immune recognition of mycobacterial cell wall products and TDM. TDM has been long recognized as one of the immunomodulatory molecules of mycobacterial cell walls. In mice, TDM induces responses that mimic certain aspects of $M$. tuberculosis infection: development of granulomatous lesions, induction of proinflammatory cytokines, decrease in the presence of serum cortisol, and enhanced procoagulant activity $(19,59,60)$. TDM has also been implicated in enhancing survival of mycobacteria by its ability to inhibit phagosome-lysosome fusion and trafficking events during 
infection (23). Although several studies have demonstrated these properties of mycobacterial TDM, the molecular mechanisms underlying these responses are not yet completely understood. Prior work has identified the role of lipid antigen presenting molecule CD1d and the C5a receptor in establishment and organization of TDM-induced granuloma formation in mice $(61,62)$. Mice deficient in either of these molecules developed edematous dysregulated granulomatous responses and also elaborated higher levels of proinflammatory cytokines in response to TDM administration. While M. tuberculosis or its cell wall components have been shown to activate macrophages via TLRs, principally TLR2 and TLR4, a recent study demonstrates that TDM-induced activation of macrophages is independent of both of these TLRs (63). However, an active role of MyD88, an adaptor for TLR- and IL-1 receptor-mediated signaling, was reported in TDM-induced cell recruitment and cytokine production, suggesting the role of a receptor other than TLR2 or TLR4 in recognition of TDM. Our present and prior works clearly establish a role for TDM and cyclopropane modification of mycolic acids of TDM in M. tuberculosis-mediated immunomodulation and pathogenesis. Identification of a cellular receptor that recognizes TDM as a whole and can distinguish cyclopropane modifications of mycolic acids on TDM is a critical area of investigation for understanding the immunopathogenesis of $M$. tuberculosis infection and its control by cell envelope lipids.

\section{Methods}

Media, strains, and culture conditions. All M. tuberculosis strains were grown in Middlebrook 7H9 liquid media supplemented with 10\% OADC (oleic acid, albumin, dextrose, catalase), $0.5 \%$ glycerol, and $0.05 \%$ Tween- 80 as well as $50 \mu \mathrm{g} / \mathrm{ml}$ of hygromycin and $20 \mu \mathrm{g} / \mathrm{ml}$ of kanamycin when required. For growth in solid media, Middlebrook 7H10 agar supplemented with $10 \%$ $\mathrm{OADC}$ and $0.5 \%$ glycerol was used, and plates were incubated at $37^{\circ} \mathrm{C}$ with $5 \% \mathrm{CO}_{2}$. An animal-passaged (minimally cultured in vitro) strain of $M$. tuberculosis Erdman was used as the WT strain for this study. The M. tuberculosis $\triangle c m a A 2$ mutant and the complemented strain ( $\triangle c m a A 2$ with a single copy of $c m a A 2$ under its native promoter) have been previously described (30).

RAW 264.7 cells and L929 cells were obtained from ATCC and were cultured in DMEM and RPMI-1640, respectively, supplemented with $10 \%$ FBS, L-glutamine, Pen-Strep, $50 \mu \mathrm{g} / \mathrm{ml}$ gentamicin HEPES, and 2-mercaptoethanol (Invitrogen). All culture media and cells were tested for LPS by the limulus amebocyte assay QCL-1000 (Cambrex) and were below the limit of detection of the assay. All cell lines and tissue culture reagents were routinely tested for mycoplasma contamination using a PCR-based assay, as described previously (64).

Purification of TDM from M. tuberculosis. TDM was purified from M. tuberculosis grown in liquid media. Cells were harvested by centrifugation and autoclaved. Autoclaved pellets were weighed and sonicated in chloroform/ methanol ( $4: 1, \mathrm{vol} / \mathrm{vol})$ for 15 minutes on ice. Water was added (1/20 total volume), and the organic phase was collected. The aqueous phase was sequentially extracted with chloroform/methanol (3:1 and 2:1, vol/vol) and the organic phases combined and evaporated completely. The dried pellet was dissolved in chloroform/methanol (3:1), and insoluble material was removed by centrifugation. The supernatant was evaporated to dryness and redissolved with acetone. The acetone insoluble phase containing TDM was collected by centrifugation. The TDM fraction was precipitated from chloroform by dropwise addition of methanol at $4{ }^{\circ} \mathrm{C}$ to a final ratio of 1:2 chloroform $/ \mathrm{methanol}$ ( $\mathrm{vol} / \mathrm{vol}$ ). This precipitate was dissolved in tetrahydrofuran and reprecipitated by dropwise addition of methanol at $4{ }^{\circ} \mathrm{C}$ to a final ratio of $1: 2$ tetrahydrofuran $/ \mathrm{methanol}(\mathrm{vol} / \mathrm{vol})$. The precipitated TDM fraction was then dissolved in chloroform/acetone (8:2, vol/vol), loaded onto a column of silica gel, and eluted with chloroform/methanol $(9: 1, \mathrm{vol} / \mathrm{vol})$. The final product was weighed, and the purity and quantity were examined by TLC using $10 \times 10 \mathrm{~cm}$ HPTLC plates (Alltech Associates Inc.) developed with chloroform/methanol/water (90:10:1, vol/vol/vol). Products were visualized by spraying with $20 \%$ sulfuric acid in ethanol and charring for 15 minutes at $110^{\circ} \mathrm{C}$.

Response of macrophages to TDM. Purified TDM was used to stimulate either RAW 264.7 cells or bone marrow-derived macrophages as described (18). Briefly, TDM was suspended at a concentration of $1 \mathrm{mg} / \mathrm{ml}$ in isopropanol, sonicated for 5 minutes in a bath sonicator (Model 3510, Branson Ultrasonic Corporation), incubated at $60^{\circ} \mathrm{C}$ for 10 minutes, and again sonicated for 5 minutes. The resulting solution was layered onto 24 -well tissue culture plates at the indicated concentrations and incubated at $37^{\circ} \mathrm{C}$ in order to ensure complete evaporation of the solvent. Control wells were layered with solvent without TDM and incubated at $37^{\circ} \mathrm{C}$. To this layer of TDM, either RAW 264.7 cells or bone marrow-derived macrophages were added at a concentration of $1 \times 10^{6}$ cells in $100 \mu \mathrm{l}$ of medium and incubated at $37^{\circ} \mathrm{C}$. At various time intervals, as indicated, after stimulation, supernatants were collected for analysis of cytokine production by using the commercial ELISA DuoSet kit (BD Biosciences) according to the manufacturer's recommendations. Intracellular cytokine staining was performed with the Cytofix/Cytoperm system (BD Biosciences) according to the manufacturer's instructions. Antibodies used were FITC-labeled anti-CD11b (Mac-1) and APC-labeled anti-TNF- $\alpha$ antibody (BD Biosciences), and CD11 $\mathrm{b}^{+}$cells were analyzed for expression of TNF- $\alpha$ in a BD LSR flow cytometer (BD Biosciences). Each analysis was performed on $25,000 \mathrm{CD}_{11} \mathrm{~b}^{+}$cells.

Isolation of bone marrow macrophages. Marrow cells were isolated from both hind limbs of mice and cultured at a concentration of $2-5 \times 10^{6}$ cells per ml in RPMI- 1640 containing 20\% FBS and 30\% L929 cell supernatant at $37^{\circ} \mathrm{C}$ in Petri dishes (OPTILUX; BD Biosciences - Discovery Labware). After 2 days, the plates were washed with sterile HBSS in order to remove the nonadherent cells. The adherent population was further incubated in macrophage growth media (RPMI $1640+20 \%$ FBS $+30 \%$ L929 cell supernatant) for 3-4 days, following which the cells were harvested.

Preparation of inoculum and infection of macrophages. Prior to infection with mycobacteria, bone marrow-derived macrophages were seeded at a concentration of $2 \times 10^{5}$ cells per well in a 24-well tissue culture dish in RPMI 1640 medium containing $10 \% \mathrm{FBS}$, without antibiotics, and incubated at $37^{\circ} \mathrm{C}$ in an atmosphere of $5 \% \mathrm{CO}_{2}$ for $16-18$ hours. The mycobacterial strains for infection of macrophages were cultured in Middlebrook $7 \mathrm{H} 9$ broth at $37^{\circ} \mathrm{C}$ to mid-log phase of growth $\left(\mathrm{A}_{600}\right.$ of $\left.0.5-0.8\right)$. The cells were harvested by centrifugation at $3,000 \mathrm{~g}$ and washed twice with PBS containing $0.05 \%$ Tween- 80 in order to remove excess media components. The cells were resuspended in PBS/Tween-80, sonicated for 5 seconds to disperse clumps, and adjusted to a concentration of $1 \times 10^{7} / \mathrm{ml}$ based on the $\mathrm{A}_{600}$, using OD1 $=5 \times 10^{8} \mathrm{CFUs} / \mathrm{ml}$. Macrophages were infected at an MOI of 5 for 6 hours at $37^{\circ} \mathrm{C}$. The cells were washed twice with sterile HBSS in order to remove extracellular bacteria. The levels of secreted cytokines in the culture supernatants were measured by ELISA.

Infection of mice by mycobacteria. Six- to eight-week old C57BL/6J or BALB/C mice obtained from Jackson Laboratory and maintained on standard feed and specific pathogen-free conditions were infected either aerogenically or intravenously with mycobacterial strains. TNF- $\alpha$-deficient and IFN- $\gamma-$ deficient mice were on the C57BL/6J background. SCID mice were on the BALB/C background. For aerosol infections, a Middlebrook inhalation exposure system (Glas-Col) was used according to procedures approved by the Memorial Sloan-Kettering Institutional Animal Care and Use Committee. Animal care and use for these experiments was approved by the Institutional Animal Care and Use Committee of the Research Animal Resource Center of Memorial Sloan Kettering Cancer Center. The mycobacterial 
inocula for murine infection were prepared as described earlier for infection of macrophages. M. tuberculosis cells were suspended at a concentration of 4 $\times 10^{8} \mathrm{CFUs}$ in $10 \mathrm{ml}$ of sterile distilled water. Mice were infected with a volume of suspension and exposure time calibrated to deliver approximately 100 CFUs per animal. For intravenous infections, mice were injected via tail vein with $200 \mu \mathrm{l}$ of mycobacterial suspension containing either $10^{5}$ or $10^{6}$ mycobacteria. The extent of infection was estimated by plating the lung homogenates of animals sacrificed at 24 hours after aerosol infection, and inocula were equal for all bacterial strains. At various time intervals after infection, the lungs and spleen were homogenized in PBS and 0.05\% Tween80 , and serial dilutions were plated onto Middlebrook $7 \mathrm{H} 10$ agar; colonies were enumerated after incubation at $37^{\circ} \mathrm{C}$ in a $5 \% \mathrm{CO}_{2}$ atmosphere. For histopathological analysis, liver lobes were removed and fixed with $10 \%$ formalin. The sections were paraffin embedded and stained with H\&E. The areas of granulomatous inflammation were calculated by digital image processing using OpenLab software (version 4.0.3; Improvision). For statistical analysis of granuloma size, 14-21 independent liver granulomas 2 weeks after intravenous infection were analyzed for total area, total nucleated cell number, and number of lymphocytes, using visual identification of size and nuclear morphology. Mean areas of the granulomatous inflammation in the different groups were compared by 2-tailed Student's $t$ test.

Delipidation and lipid reconstitution of mycobacteria. Delipidation of bacilli was performed by using petroleum ether, using a modification of published methods (22) and as described previously (18). Following two 5-minute extractions with petroleum ether, delipidated cells were centrifuged and suspended in PBS/0.05\% Tween-80 for infection of macrophage cultures. Reconstitution of lipids was done by incubating delipidated cells with the petroleum ether extracts of mycobacterial cultures of identical cell number for 30 minutes at $25^{\circ} \mathrm{C}$, following which cells were harvested by centrifugation and suspended in PBS/0.05\% Tween 80. Macrophage cultures were infected with the native, delipidated, and reconstituted bacilli at an MOI of 5 as described earlier.

Statistics. For statistical significance between 2 groups, the individual values of both groups were analyzed by using the 2-tailed Student's $t$ test. A $P$ value of less than 0.05 was considered significant.

\section{Acknowledgments}

This work was supported by a grant from the NIH (AI53417 to M.S. Glickman) and a New Scholars Award from the Ellison Medical Foundation (M.S. Glickman).

Received for publication November 4, 2005, and accepted in revised form March 14, 2006.

Address correspondence to: Michael S. Glickman, Memorial SloanKettering Cancer Center, Box 9, 1275 York Avenue, New York, New York 10021, USA. Phone: (212) 639-3191; Fax: (646) 422-2124; E-mail: glickmam@mskcc.org.
1. Monack, D.M., Mueller, A., and Falkow, S. 2004. Persistent bacterial infections: the interface of the pathogen and the host immune system. Nat. Rev. Microbiol. 2:747-765.

2. Smith, I. 2003. Mycobacterium tuberculosis pathogenesis and molecular determinants of virulence. Clin. Microbiol. Rev. 16:463-496.

3. Hingley-Wilson, S.M., Sambandamurthy, V.K., and Jacobs, W.R., Jr. 2003. Survival perspectives from the world's most successful pathogen, Mycobacterium tuberculosis. Nat. Immunol. 4:949-955.

4. Cosma, C.L., Sherman, D.R., and Ramakrishnan, L. 2003. The secret lives of the pathogenic mycobacteria. Annu. Rev. Microbiol. 57:641-676.

5. Clark-Curtiss, J.E., and Haydel, S.E. 2003. Molecular genetics of Mycobacterium tuberculosis pathogenesis. Annu. Rev. Microbiol. 57:517-549.

6. Barry, C.E., 3rd, et al. 1998. Mycolic acids: structure, biosynthesis and physiological functions. Prog. Lipid Res. 37:143-179.

7. Brennan, P.J., and Nikaido, H. 1995. The envelope of mycobacteria. Annu. Rev. Biochem. 64:29-63.

8. Minnikin, D.E., Kremer, L., Dover, L.G., and Besra, G.S. 2002. The methyl-branched fortifications of Mycobacterium tuberculosis. Chem. Biol. 9:545-553.

9. Camacho, L.R., Ensergueix, D., Perez, E., Gicquel, B., and Guilhot, C. 1999. Identification of a virulence gene cluster of Mycobacterium tuberculosis by signature-tagged transposon mutagenesis. Mol. Microbiol. 34:257-267.

10. Cox, J.S., Chen, B., McNeil, M., and Jacobs, W.R., Jr. 1999. Complex lipid determines tissue-specific replication of Mycobacterium tuberculosis in mice. Nature. 402:79-83.

11. Dubnau, E., et al. 2000. Oxygenated mycolic acids are necessary for virulence of Mycobacterium tuberculosis in mice. Mol. Microbiol. 36:630-637.

12. Gao, L.Y., et al. 2003. Requirement for kasB in Mycobacterium mycolic acid biosynthesis, cell wall impermeability and intracellular survival: implications for therapy. Mol. Microbiol. 49:1547-1563.

13. Glickman, M.S., Cox, J.S., and Jacobs, W.R., Jr. 2000. A novel mycolic acid cyclopropane synthetase is required for cording, persistence, and virulence of Mycobacterium tuberculosis. Mol. Cell. 5:717-727.

14. Makinoshima, H., and Glickman, M.S. 2005.
Regulation of Mycobacterium tuberculosis cell envelope composition and virulence by intramembrane proteolysis. Nature. 436:406-409.

15. Reed, M.B., et al. 2004. A glycolipid of hypervirulent tuberculosis strains that inhibits the innate immune response. Nature. 431:84-87.

16. Camacho, L.R., et al. 2001. Analysis of the phthiocerol dimycocerosate locus of Mycobacterium tuberculosis. Evidence that this lipid is involved in the cell wall permeability barrier. J. Biol. Chem. 276:19845-19854

17. Daffe, M., Papa, F., Laszlo, A., and David, H.L. 1989. Glycolipids of recent clinical isolates of Mycobacterium tuberculosis: chemical characterization and immunoreactivity. J. Gen. Microbiol. 135:2759-2766.

18. Rao, V., Fujiwara, N., Porcelli, S.A., and Glickman, M.S. 2005. Mycobacterium tuberculosis controls host innate immune activation through cyclopropane modification of a glycolipid effector molecule. J. Exp. Med. 201:535-543.

19. Behling, C.A., Bennett, B., Takayama, K., and Hunter, R.L. 1993. Development of a trehalose 6,6'-dimycolate model which explains cord formation by Mycobacterium tuberculosis. Infect. Immun. 61:2296-2303.

20. Behling, C.A., Perez, R.L., Kidd, M.R., Staton, G.W., Jr., and Hunter, R.L. 1993. Induction of pulmonary granulomas, macrophage procoagulant activity, and tumor necrosis factor-alpha by trehalose glycolipids. Ann. Clin. Lab. Sci. 23:256-266.

21. Yamagami, H., et al. 2001. Trehalose 6,6'-dimycolate (cord factor) of Mycobacterium tuberculosis induces foreign-body- and hypersensitivity-type granulomas in mice. Infect. Immun. 69:810-815.

22. Indrigo, J., Hunter, R.L., Jr., and Actor, J.K. 2002. Influence of trehalose $6,6^{\prime}$-dimycolate (TDM) during mycobacterial infection of bone marrow macrophages. Microbiology. 148:1991-1998.

23. Indrigo, J., Hunter, R.L., Jr., and Actor, J.K. 2003. Cord factor trehalose 6,6'-dimycolate (TDM) mediates trafficking events during mycobacterial infection of murine macrophages. Microbiology. 149:2049-2059.

24. Asselineau, J., Bloch, H., and Lederer, E. 1953. A toxic lipid component of the tubercle bacillus (cord factor). III. Occurrence and distribution in various bacterial extracts. Am. Rev. Tuberc. 67:853-858.

25. Noll, H., and Bloch, H. 1953. A toxic lipid component of the tubercle bacillus (cord factor). II. Occurrence in chloroform extracts of young and older bacterial cultures. Am. Rev. Tuberc. 67:828-852.

26. Bloch, H., Sorkin, E., and Erlenmeyer, H. 1953. A toxic lipid component of the tubercle bacillus (cord factor). I. Isolation from petroleum ether extracts of young bacterial cultures. Am. Rev. Tuberc. 67:629-643.

27. Glickman, M.S., and Jacobs, W.R., Jr. 2001. Microbial pathogenesis of Mycobacterium tuberculosis: dawn of a discipline. Cell. 104:477-485.

28. Yuan, Y., Mead, D., Schroeder, B.G., Zhu, Y., and Barry, C.E., 3rd. 1998. The biosynthesis of mycolic acids in Mycobacterium tuberculosis. Enzymatic methyl(ene) transfer to acyl carrier protein bound meromycolic acid in vitro. J. Biol. Chem. 273:21282-21290.

29. Yuan, Y., Crane, D.C., Musser, J.M., Sreevatsan, S., and Barry, C.E., 3rd. 1997. MMAS-1, the branch point between cis- and trans-cyclopropane-containing oxygenated mycolates in Mycobacterium tuberculosis. J. Biol. Chem. 272:10041-10049.

30. Glickman, M.S., Cahill, S.M., and Jacobs, W.R., Jr. 2001. The Mycobacterium tuberculosis cmaA2 gene encodes a mycolic acid trans cyclopropane synthetase. J. Biol. Chem. 276:2228-2233.

31. Glickman, M.S. 2003. The $m m a A 2$ gene of Mycobacterium tuberculosis encodes the distal cyclopropane synthase of the alpha-mycolic acid. J. Biol. Chem. 278:7844-7849.

32. Dubnau, E., et al. 1997. Mycobacterium bovis BCG genes involved in the biosynthesis of cyclopropyl keto- and hydroxy-mycolic acids. Mol. Microbiol. 23:313-322.

33. Cronan, J.E., Jr. 2002. Phospholipid modifications in bacteria. Curr. Opin. Microbiol. 5:202-205.

34. Bao, X., Thelen, J.J., Bonaventure, G., and Ohlrogge, J.B. 2003. Characterization of cyclopropane fattyacid synthase from Sterculia foetida. J. Biol. Chem. 278:12846-12853.

35. Bao, X., Katz, S., Pollard, M., and Ohlrogge, J. 2002. Carbocyclic fatty acids in plants: biochemical and molecular genetic characterization of cyclopropane fatty acid synthesis of Sterculiafoetida. Proc. Natl. Acad. Sci. U. S. A. 99:7172-7177. 
36. Bohannon, M.B., and Kleiman, R. 1978. Cyclopropene fatty acids of selected seed oils from bombacaceae, malvaceae, and sterculiaceae. Lipids. 13:270-273.

37. Ando, M., Yoshimatsu, T., Ko, C., Converse, P.J., and Bishai, W.R. 2003. Deletion of Mycobacterium tuberculosis sigma factor $E$ results in delayed time to death with bacterial persistence in the lungs of aerosolinfected mice. Infect. Immun. 71:7170-7172.

38. Steyn, A.J., et al. 2002. Mycobacterium tuberculosis WhiB3 interacts with RpoV to affect host survival but is dispensable for in vivo growth. Proc. Natl. Acad. Sci. U. S. A. 99:3147-3152.

39. Manca, C., et al. 1999. Mycobacterium tuberculosis CDC1551 induces a more vigorous host response in vivo and in vitro, but is not more virulent than other clinical isolates. J. Immunol. 162:6740-6746.

40. Calamita, H., et al. 2005. The Mycobacterium tuberculosis SigD sigma factor controls the expression of ribosome-associated gene products in stationary phase and is required for full virulence. Cell. Microbiol. 7:233-244.

41. Sun, R., et al. 2004. Mycobacterium tuberculosis ECF sigma factor sigC is required for lethality in mice and for the conditional expression of a defined gene set. Mol. Microbiol. 52:25-38.

42. Flynn, J.L., et al. 1995. Tumor necrosis factor-alpha is required in the protective immune response against Mycobacterium tuberculosis in mice. Immunity. 2:561-572.

43. Keane, J., et al. 2001. Tuberculosis associated with infliximab, a tumor necrosis factor alpha-neutralizing agent. N. Engl. J. Med. 345:1098-1104.

44. Flynn, J.L., and Chan, J. 2005. What's good for the host is good for the bug. Trends Microbiol. 13:98-102.

45. Chan, J., and Flynn, J. 2004. The immunological aspects of latency in tuberculosis. Clin. Immunol. 110:2-12.

46. Tufariello, J.M., Chan, J., and Flynn, J.L. 2003. Latent tuberculosis: mechanisms of host and bacil- lus that contribute to persistent infection. Lancet Infect. Dis. 3:578-590.

47. Bean, A.G., et al. 1999. Structural deficiencies in granuloma formation in TNF gene-targeted mice underlie the heightened susceptibility to aerosol Mycobacterium tuberculosis infection, which is not compensated for by lymphotoxin. J. Immunol. 162:3504-3511.

48. Flynn, J.L., et al. 1993. An essential role for interferon gamma in resistance to Mycobacterium tuberculosis infection. J. Exp. Med. 178:2249-2254.

49. Parish, T., et al. 2003. Deletion of two-component regulatory systems increases the virulence of $\mathrm{Myco}$ bacterium tuberculosis. Infect. Immun. 71:1134-1140.

50. McAdam, R.A., et al. 2002. Characterization of a Mycobacterium tuberculosis $\mathrm{H} 37 \mathrm{Rv}$ transposon library reveals insertions in 351 ORFs and mutants with altered virulence. Microbiology. 148:2975-2986.

51. Shimono, N., et al. 2003. Hypervirulent mutant of Mycobacterium tuberculosis resulting from disruption of the mce1 operon. Proc. Natl. Acad. Sci. U. S. A. 100:15918-15923.

52. Moreira, A.L., et al. 2002. Mycobacterial antigens exacerbate disease manifestations in Mycobacterium tuberculosis-infected mice. Infect. Immun. 70:2100-2107.

53. Ehlers, S., Kutsch, S., Ehlers, E.M., Benini, J., and Pfeffer, K. 2000. Lethal granuloma disintegration in mycobacteria-infected TNFRp $55^{-/-}$mice is dependent on T cells and IL-12. J. Immunol. 165:483-492.

54. Bekker, L.G., et al. 2000. Immunopathologic effects of tumor necrosis factor alpha in murine mycobacterial infection are dose dependent. Infect. Immun. 68:6954-6961.

55. Fortune, S.M., et al. 2004. Mycobacterium tuberculosis inhibits macrophage responses to IFN-gamma through myeloid differentiation factor 88-dependent and -independent mechanisms. J. Immunol.
172:6272-6280

56. Saunders, B.M., Briscoe, H., and Britton, W.J. 2004. $\mathrm{T}$ cell-derived tumour necrosis factor is essential, but not sufficient, for protection against $\mathrm{Myco}$ bacterium tuberculosis infection. Clin. Exp. Immunol. 137:279-287.

57. Chang, Y.Y., and Cronan, J.E., Jr. 1999. Membrane cyclopropane fatty acid content is a major factor in acid resistance of Escherichia coli. Mol. Microbiol. 33:249-259.

58. Rachman, H., et al. 2006. Unique transcriptome signature of Mycobacterium tuberculosis in pulmonary tuberculosis. Infect. Immun. 74:1233-1242.

59. Actor, J.K., et al. 2002. Mycobacterial glycolipid cord factor trehalose 6,6'-dimycolate causes a decrease in serum cortisol during the granulomatous response. Neuroimmunomodulation. 10:270-282.

60. Perez, R.L., Roman, J., Staton, G.W., Jr., and Hunter, R.L. 1994. Extravascular coagulation and fibrinolysis in murine lung inflammation induced by the mycobacterial cord factor trehalose-6,6'-dimycolate. Am. J. Respir. Crit. Care Med. 149:510-518.

61. Borders, C.W., et al. 2005. Requisite role for complement C5 and the C5a receptor in granulomatous response to mycobacterial glycolipid trehalose 6,6'dimycolate. Scand. J. Immunol. 62:123-130.

62. Guidry, T.V., et al. 2004. Failure of CD1D ${ }^{-/-}$mice to elicit hypersensitive granulomas to mycobacterial cord factor trehalose 6,6'-dimycolate. J. Interferon Cytokine Res. 24:362-371.

63. Geisel, R.E., Sakamoto, K., Russell, D.G., and Rhoades, E.R. 2005. In vivo activity of released cell wall lipids of Mycobacterium bovis bacillus CalmetteGuerin is due principally to trehalose mycolates. J. Immunol. 174:5007-5015.

64. Tang, J., Hu, M., Lee, S., and Roblin, R. 2000. A polymerase chain reaction based method for detecting mycoplasma/acholeplasma contaminants in cell culture. J. Microbiol. Methods. 39:121-126. 